\title{
The Free-Energy Landscape of Clusters of Attractive Hard Spheres
}

\section{Citation}

Meng, Guangnan, Natalie Arkus, Michael P. Brenner, and Vinothan N. Manoharan. 2010. The Free-Energy Landscape of Clusters of Attractive Hard Spheres. Science 327 (5965): 560-63. doi:10.1126/science.1181263

\section{Published Version}

doi:10.1126/science. 1181263

\section{Permanent link}

http://nrs.harvard.edu/urn-3:HUL.InstRepos:11859334

\section{Terms of Use}

This article was downloaded from Harvard University's DASH repository, and is made available under the terms and conditions applicable to Other Posted Material, as set forth at http:// nrs.harvard.edu/urn-3:HUL.InstRepos:dash.current.terms-of-use\#LAA

\section{Share Your Story}

The Harvard community has made this article openly available.

Please share how this access benefits you. Submit a story.

\section{Accessibility}




\title{
The Free Energy Landscape of Clusters of Attractive Hard Spheres*
}

\author{
Guangnan Meng ${ }^{1}$, Natalie Arkus ${ }^{2}$, Michael P. Brenner ${ }^{2}$ and \\ Vinothan N. Manoharan ${ }^{1,2}$ \\ ${ }^{1}$ Department of Physics, Harvard University, Cambridge MA \\ ${ }^{2}$ Harvard School of Engineering and Applied Sciences, Harvard University, \\ Cambridge MA 02138 USA
}

One sentence summary: Experiments reveal that small clusters of hard spheres with short-range attractions favor equilibrium structures determined by geometrical rules.

The study of clusters has provided the most tangible link between local geometry and bulk condensed matter. But experiments have not yet systematically explored the thermodynamics of even the smallest clusters. Here we present experimental measurements of the structures and free energies of colloidal clusters in which the particles act as hard spheres with short-range attractions. We find that highly symmetric clusters are strongly suppressed by rotational entropy, while the most stable clusters have anharmonic vibrational modes or extra bonds. Many of these are subsets of closepacked lattices. As the number of particles increases from 6 to 10 we observe the emergence of a complex free energy landscape with a small number of ground states and many local minima.

An isolated system of 10 interacting atoms or molecules will in general adopt a structure that differs in symmetry and average energy from that of a bulk liquid, solid, or even a system containing 100 particles. Yet the study of such small clusters has shed light on a wide variety of phenomena in condensed matter physics and physical chemistry. Since Frank first predicted (1) that icosahedral short-range order

\footnotetext{
${ }^{*}$ This is the authors version of the work. It is posted here by permission of the AAAS for personal use, not for redistribution. The definitive version was published in Science volume 327 on January 29, 2010, DOI: 10.1126/science. 1181263
} 
would be a hallmark of liquid structure, the study of small cluster geometry has provided key insights into the frustration underlying non-equilibrium phenomena such as nucleation and the glass transition $(2,3,4)$. Experimental studies (5, 6) have confirmed this approach through the discovery of local cluster-like order in bulk liquids and glasses, with recent results (7) suggesting that structural arrest in condensed phases may be related to geometrical constraints at the scale of a few particles.

There remain many unresolved questions about cluster geometry and the connection to bulk behavior. Although experiments and simulations have determined the minimum potential energy clusters for various interactions $(8,9,10)$, the likelihood of observing a particular cluster structure depends on its free energy $(11,12)$. What cluster structures are favored by entropy? And how does the competition between potential energy and entropy evolve as the number of particles $N$ approaches the bulk limit? Experiments on atomic clusters have not systematically explored these questions; they are limited by short cluster lifetimes, non-equilibrium conditions, and the difficulties of obtaining real-space structures of individual clusters in free space (13).

We report experimental results for the structures and free energies of small equilibrium clusters as a function of $N$, with $N \leq 10$. The experimental system is described in Fig. 1. We use colloidal particles rather than atoms because we can precisely control the interactions and directly observe the three-dimensional (3D) structures of the clusters using optical microscopy. To a good approximation our particles act as "sticky" hard spheres, arguably the simplest non-trivial interaction that leads to clustering. The attraction arises from a depletion interaction with a range of about 1.05 times the particle diameter and a depth of about $4 k_{B} T$. Because the pair potential is short-ranged, the total potential energy $U$ of a given structure is well approximated by $U=C U_{m}$, where $C$ is the number of contacts or depletion "bonds" and $U_{m}$ the depth of the pair potential (14). While in bulk these particles form a gel, the range and depth of the interaction are consistent with an equilibrium phase diagram showing a fluid-crystal transition. (17).

We create clusters by isolating small numbers of polystyrene (PS) microspheres in cylindrical microwells filled with water and poly $(N$-isopropylacrylamide) (polyNIPAM) nanoparticles, which cause the depletion interaction. We chemically functionalize the microwells so that particles cannot stick to the surfaces. This allows 3D clusters to form in the middle of the wells, unaffected by the boundaries. After the clusters reach equilibrium, we use optical microscopy to observe the cluster structures, and we collect statistics by scanning through the microwell plate, which contains thousands of isolated clusters. Although the number of particles per well is not controlled, we generate enough clusters at each $N \leq 10$ to measure their occurrence frequencies. We then determine the free energies from the ensemble statistics through the Boltzmann distribution: $\Delta F=-k_{B} T \ln P$, where $P$ is the probability of 

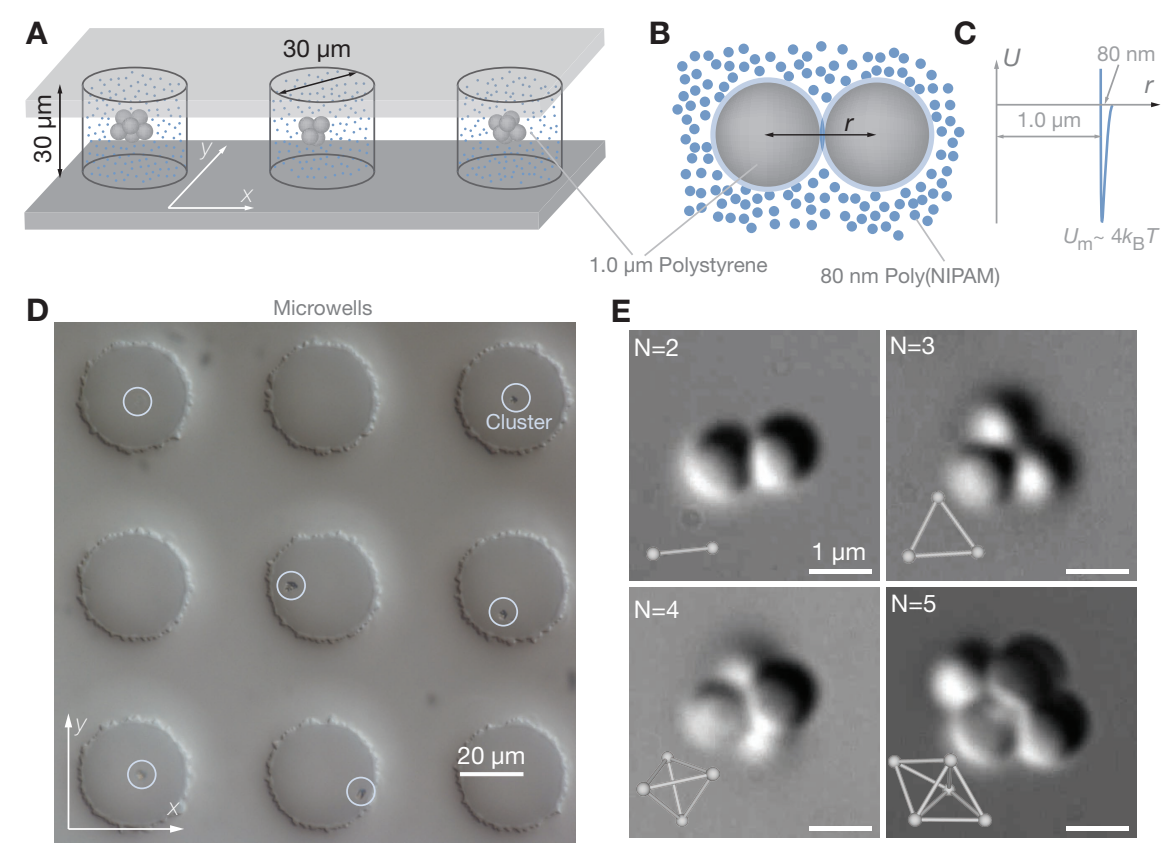

$\mathbf{E}$
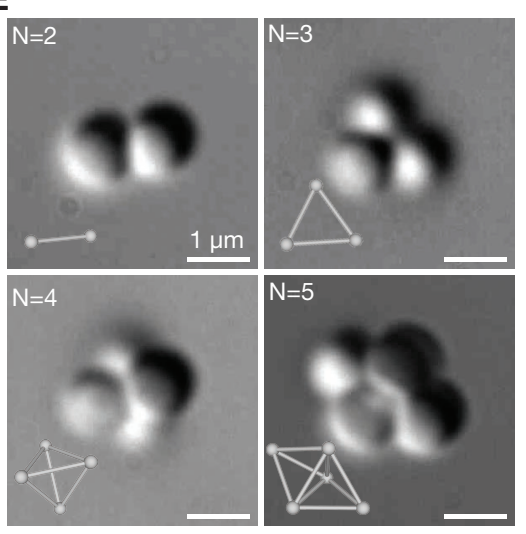

Figure 1: (A) Diagram of experimental system (14). We use lithography to make microwells with depth and diameter $30 \mu \mathrm{m}$ (see also Fig. S1). These are filled with a suspension of $1.0 \mu \mathrm{m}$ diameter polystyrene (PS) spheres and $80 \mathrm{~nm}$ poly $(N$ isopropylacrylamide) (polyNIPAM) microgel particles, which induce a depletion attraction as illustrated in (B). The number of PS particles per well varies, but the average is about 10. (C) Pair potential as estimated from the Vrij approximation to the Asakura-Oosawa potential. $(15,16)$. Because the range of the depletion attraction is less than 1/10 the PS sphere diameter, the interaction is strictly pairwise additive. (D) Optical micrograph of microwells with assembled colloidal clusters suspended inside. The circles highlight individual clusters in different microwells. There are about $10^{4}$ microwells per slide. (E) High magnification optical micrographs of colloidal clusters in microwells with $N=2,3,4,5$ particles. These are the only structures that form for $N \leq 5$. 
observing a given cluster.

We classify our clusters by comparing them to finite sphere packings. A previous theoretical study (18) enumerated the mechanically stable clusters of idealized hard spheres with infinitesimally short-ranged interactions, revealing the minima of the potential energy landscape as a function of $N$. All of the minima at each $N \leq 9$ have the same potential energy, a situation not observed with longer-ranged potentials (19). Here we explore the structures and probabilities of these packings at finite temperature, which allows us to map the free energy landscape $(20,21,11)$. All the observed cluster structures agree with the theoretical predictions. For example, for $N<6$ we observe one unique structure for each $N$ : a dimer for $N=2$, trimer for $N=3$, tetrahedron for $N=4$ and triangular dipyramid for $N=5$. The optical micrographs in Fig. 1 show the structures of the smallest clusters.

The first interesting case is $N=6$. Here we observe two structures (Fig. 2 and Fig. S2), both with $C=12$ contacts and therefore equivalent potential energy. The first is the octahedron, a Platonic solid. The second we call a "polytetrahedron." It consists of a triangular dipyramid with a third tetrahedron added to one of the faces. We observe transitions between the two states on time scales of minutes, indicating that the system is at equilibrium (Movie S1).

Even though these two structures have the same potential energies, the polytetrahedron occurs about 20 times more often than the octahedron, implying a free energy difference of about $3 k_{B} T$. This difference can be attributed only to entropy. As shown in Fig. 2, the measured probabilities for the two structures agree well with theoretical calculations based on standard approximations for the rotational and vibrational entropies in the classical limit (14).

Notably, the rotational entropy makes the largest contribution to the free energy difference between the two structures (Fig. S2). The rotational partition function is related to two geometrical quantities: the number of orientations, which is proportional to the moment of inertia, and the rotational symmetry of the cluster, or, alternatively, the number of ways one can assemble the same cluster by permuting particle labels (22). Formally, the ratio of the permutational degeneracies of two clusters is inversely proportional to the ratio of their symmetry numbers (23). This permutational degeneracy accounts for a factor of 12 in the polytetrahedron:octahedron probability ratio. The remaining factor of 2 comes from the differences in the moments of inertia and the vibrational entropies.

This illustrates a general rule for clusters with short-range attractions: among clusters with the same potential energy, highly symmetric structures are highly unfavorable at equilibrium. By contrast, for the longer-ranged Lennard-Jones 6-12 potential the octahedron has lower potential energy than the polytetrahedron (19), so that the dominant structure depends on temperature. The dominance of the polytetrahedron in our system may have consequences for nucleation: the equilibrium phase of attractive hard spheres is a face-centered cubic (FCC) crystal (17), which 

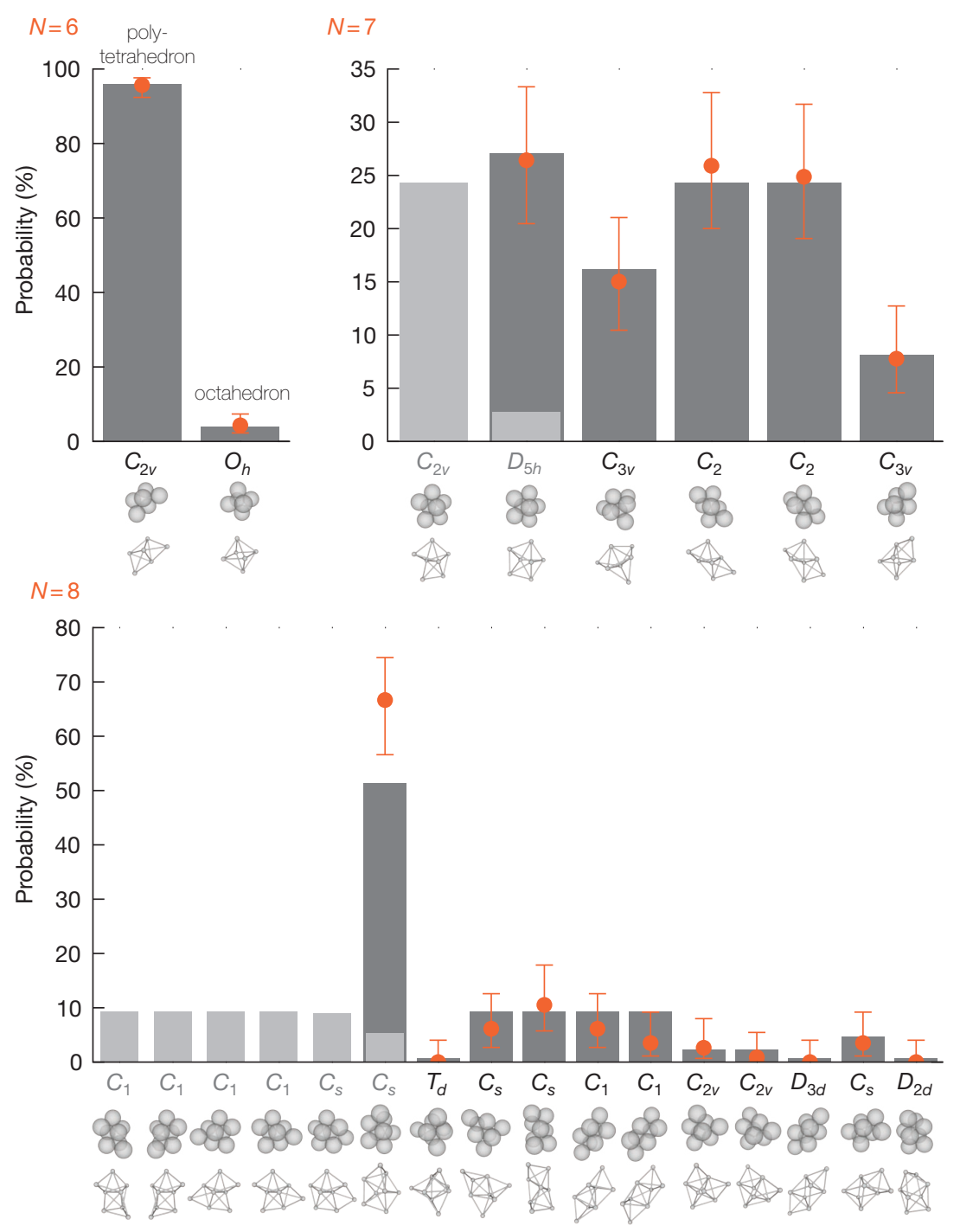

Figure 2: Comparison of experimental and theoretical (14) cluster probabilities $P$ at $N=6,7$, and 8 . Structures difficult to differentiate experimentally have been binned together at $N=7$ and $N=8$ to compare to theory. The calculated probabilities for the individual states are shown in light gray bars, and binned probabilities in dark gray. Red dots indicate the experimental measurements, with $95 \%$ confidence intervals given by the error bars (see (14) and Table S1). Renderings and point groups in Schönflies notation are shown for each structure. The number in the subscript of each symbol indicates the order of the highest rotational symmetry axis, and the letter indicates the symmetry group. The highest symmetry structures are those in $D, T$, and $O$ groups. Structures in $C_{1}$ and $C_{2}$ groups occur in chiral pairs. 
contains octahedral, not polytetrahedral, subunits.

At $N=7$ the first chiral structures arise. We observe six cluster structures, two of which are chiral enantiomers. The experimental measurements agree well with the theoretical values for the probabilities of each structure, as shown in Fig. 2.

For these small clusters, the most pronounced influence on the probabilities comes from symmetry. At $N=8$, three of the 16 different possible sphere packings never occur in the experiments. These three structures have the highest symmetry numbers, $\sigma=4,6,12$.

A few structures differ by such small changes in particle spacing that we cannot differentiate between them in our microscope. All of these are variants of pentagonal dipyramids. In a pentagonal dipyramid of 7 spheres, the top and bottom spheres of the pyramid are separated by a small gap of $\approx 0.05 d$, where $d$ is the sphere diameter. If these two spheres are brought together, a gap of $\approx 0.09 d$ opens between two of the spheres on the pentagon. Because we cannot resolve this gap in our experiments, we have binned these structures together at both $N=7$ and $N=8$. The one statistically significant discrepancy between experiment and theory occurs at $N=8$; it arises because the experimental potential has a range comparable to the gap distance. Although we account for this extra potential energy in the probability calculations, the probabilities are sensitive to the magnitude of the potential at the gap distance. If the interaction energy differs from our estimated value by only $0.1 k_{B} T$ in the gap, the theoretical calculation falls within error of the experimental value. This difference could be due to polydispersity in either the depletant or large spheres, or to another interaction such as van der Waals forces.

The probability distributions in Fig. 2 are "field guides" to the free energy landscapes at $N=6,7$, and 8 . Each structure represents a local free energy minimum, the depth of which is proportional to the probability. We note two topographical features besides the trend toward structures with low symmetry: first, the number of local minima increases dramatically with $N$, and second, the landscape is relatively flat for $N=7$ and 8 . In other words, there are many shallow minima, but no one minimum has a free energy much larger than any other.

The landscape undergoes a qualitative change for $N \geq 9$. Theoretically we expect some $\Omega=77$ structures at $N=9$ and $\Omega=393$ at $N=10$, too many to catalogue experimentally. We therefore measure only a subset of structures identified by our theoretical study (18). The subset we choose consists of clusters that fall into either of two categories: non-rigid structures, in which one of the vibrational modes is a large amplitude, anharmonic shear mode, and structures with more than $3 N-6$ bonds. Non-rigidity arises when a cluster contains half-octahedra that share at least one vertex, allowing the cluster to twist over a finite distance without breaking or forming another bond. We expect these packings to have high vibrational entropy. Structures with more than $3 N-6$ bonds can occur for $N \geq 10$. These are the expected ground states. 

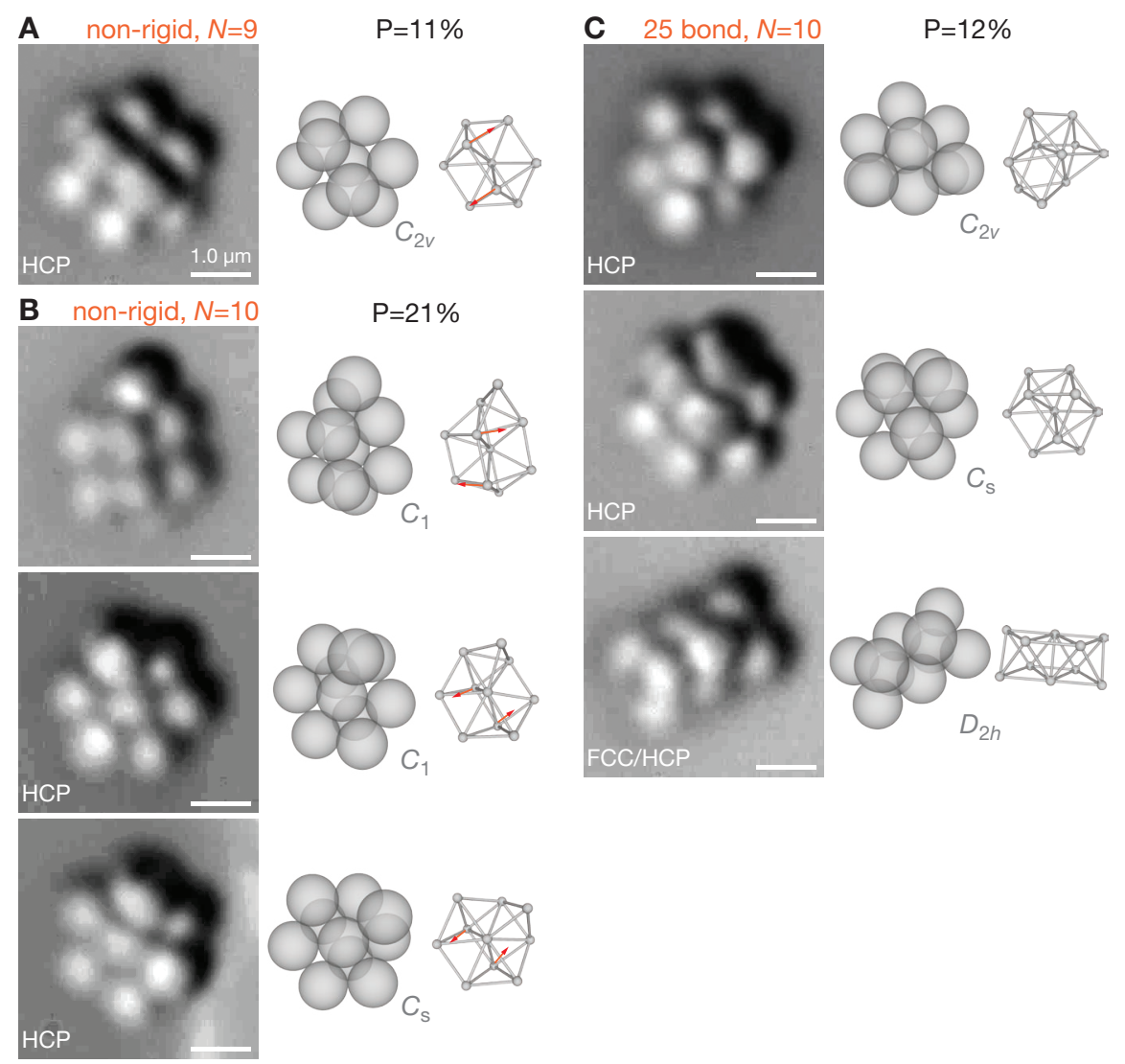

FCC/HCP

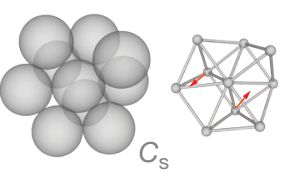

Figure 3: (A) Optical micrographs and renderings of non-rigid structures at $N=9$ (see also Movies S2 and S3) and (B) $N=10$ (Movies S4-S6). (C) structures of $3 N-5=25$ bond packings at $N=10$ (Movies S7-S9). The anharmonic vibrational modes of the non-rigid structures are shown by red arrows. Experimentally measured probabilities are listed at top. Annotations in micrographs indicate clusters corresponding to subsets of hexagonally close-packed (HCP) or face-centered cubic (FCC) lattices. 
Indeed, these special packings do occur with high frequency, as shown in Fig. 3 and Table S2. Because most clusters at $N=9$ and 10 have equal potential energy, low symmetry, and therefore comparable rotational entropy, we expect the average probability of any one structure in a set of $\Omega$ possible clusters to be of order $1 / \Omega$. At $N=9$ we expect an average probability of about $1 \%$, and at $N=10$, about $0.25 \%$.

By contrast, the one non-rigid structure at $N=9$ occurs with $P \approx 10 \%$. Using the theoretical $\Omega$ and the experimental $P$ we estimate that the free energy of the nonrigid structure is about $2 k_{B} T$ lower than that of an average structure at $N=9(14)$. Thus the structure is highly stable, by nearly half the free energy of an extra bond. The stabilization comes from the vibrational entropy associated with the non-rigid mode depicted in Movie S3. Our theoretical calculations (14) predict $P \approx 3 \%$, which is lower than the observed probability but higher than all other clusters at $N=$ 9. The discrepancy is due to the sensitive dependence of the vibrational partition function on the curvature of the pair potential near the minimum, a consequence of the non-rigid mode. A more precise calculation requires an accurate measurement of electrostatic effects in the experimental pair potential near the depletion well.

At $N=10$, only three of the 393 theoretically possible clusters have $3 N-5=25$ contacts, yet these occur about $10 \%$ of the time. Although we have only limited statistics for higher $N$, we continue to observe the prevalence of a few packings with $3 N-5$ or more bonds. The structures with extra bonds have combined probabilities of $20-30 \%$ at $N=11$ and $N=12$ (Table S2). Again these probabilities are large compared to $1 / \Omega$, even though in several cases the clusters have high symmetry. The potential energy gain is therefore large enough to overcome the deficiency in rotational entropy.

Perhaps the most striking feature of these clusters is that many are subsets of lattice packings, and in particular of the hexagonally close packed (HCP) lattice. The lattice packings are marked in Fig. 3. The underlying reason appears to be that both non-rigidity and extra bonds require the clusters to have octahedral subunits. The propensity for icosahedra $(8,24)$ in longer-range systems is absent in ours. We observe no icosahedra at either $N=12$ or $N=13$, presumably because neither 12-sphere or 13-sphere icosahedra are special clusters for short-range interactions: neither are non-rigid, neither have more than $3 N-6$ bonds, and both have very high symmetry numbers $(\sigma=60)$.

Using the same statistical mechanical approximations used to estimate probabilities for $N \leq 8$, we can calculate the free energies of all mechanically stable sphere packings that have been enumerated (18) up to $N=10$. This yields the free energy landscape shown in Fig. 4. We see that in general the locus of states is correlated with the rotational entropy, which is proportional to $k_{B} \ln (\sqrt{I} / \sigma)$. The only states that lie below this locus occur at $N=9$ and 10 . These correspond to either non-rigid structures or structures with extra bonds, both of which appear as deeper minima.

The diagram also reveals some new features. First, low-symmetry polytetrahe- 


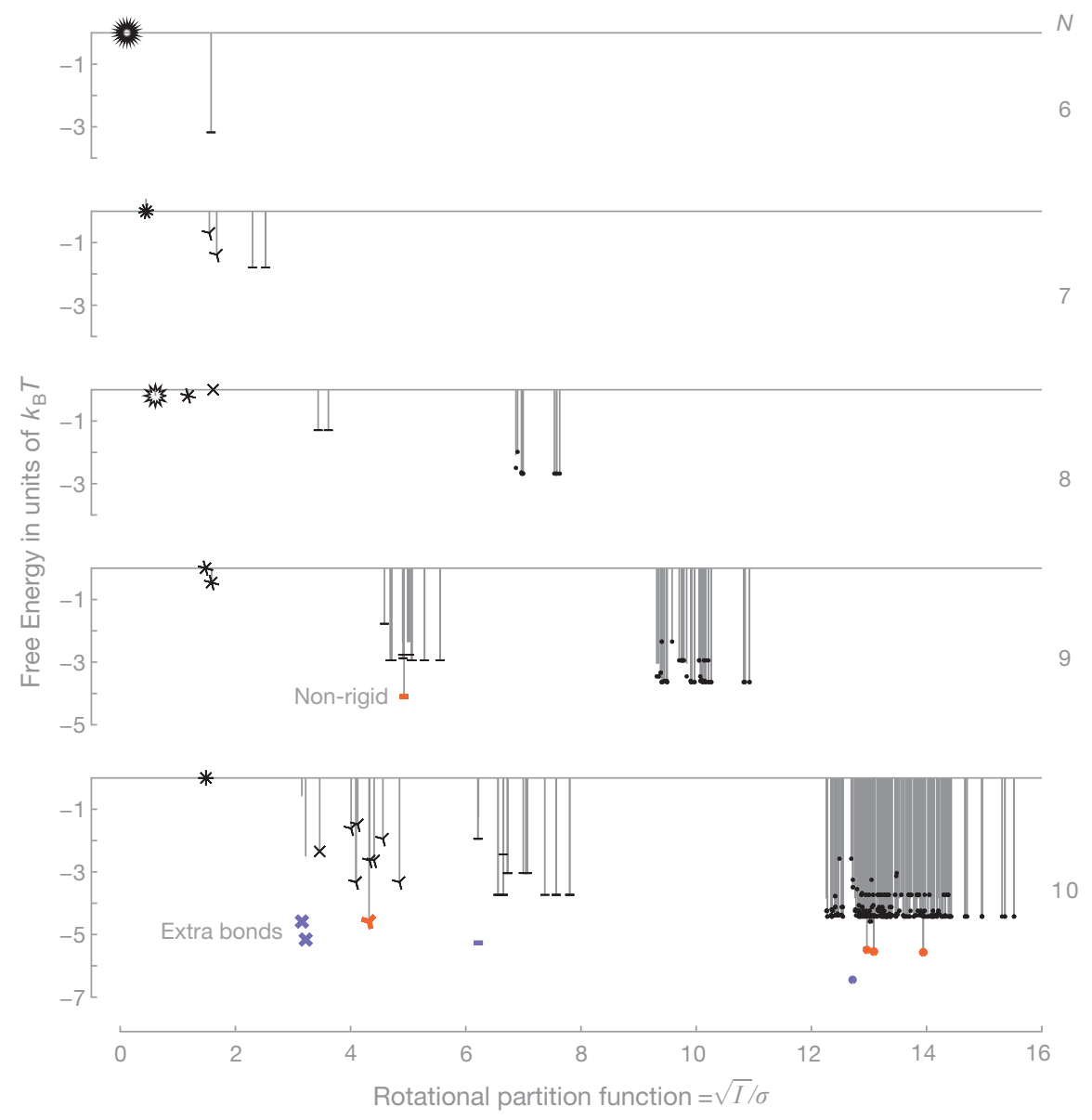

Figure 4: Calculated minima of the free energy landscape for $6 \leq N \leq 10$ (14). $\mathrm{x}$ axis is in units of the rotational partition function, where $I$ is the moment of inertia (calculated for a particle mass equal to 1 ) and $\sigma$ the rotational symmetry number. The bond strength for the calculation is $U_{m}=4 k_{B} T$. Each black symbol represents the free energy of an individual cluster. The number of spokes in each symbol indicates the symmetry number ( $\operatorname{dot}=1$, line segment $=2$, and so on). Red symbols are non-rigid structures, which first appear at $N=9$, and violet symbols have extra bonds, first appearing at $N=10$. Vertical gray lines indicate the contribution to the free energy due to rotational and vibrational entropy. The reference states are chosen to be the highest free energy states at each $N$. The general trend is for low symmetry states to be favored in proportion to their rotational entropy, $k_{B} \ln (\sqrt{I} / \sigma)$. The potential energy contribution accounts for the vertical space between the violet symbols and their gray lines. 
dral states proliferate as $N$ increases. At $N=10$, where clusters with extra bonds first appear, the absolute probability of observing these ground states is low because of the large number of low-symmetry states that lie at slightly higher free energy.

Second, the highest free energy structures for $N=6-10$ are convex deltahedra (25), polyhedra with a long history in condensed matter physics $(26,3)$. These are not always the most symmetric structures: at $N=8$ the highest free energy state is the deltahedron, a snub disphenoid, which has lower symmetry than an 8-sphere tetrahedral cluster. The convex deltahedra also happen to be the same "minimalmoment" structures formed in capillary-driven assembly of colloidal particles (27). The optimal packings under these non-equilibrium conditions therefore correspond to the least optimal packings at equilibrium.

Our results suggest that nucleation barriers and structural motifs in attractive hard sphere systems such as colloidal suspensions will be different from those in systems with longer-range potentials, which tend to favor symmetric structures at sufficiently low temperatures. For $N<9$ all of our clusters have nearly equivalent potential energy, and therefore the rotational entropy selects against symmetric structures at all temperatures. Specifically, the symmetry number and the permutational degeneracy have the greatest effect on the free energy; differences in moment of inertia do not contribute as much. Thus even if cluster rotations are hindered, as they may be in a bulk supercooled liquid, the permutational degeneracy might still influence the probability of formation. At higher $N$ the most probable structures we observe involve combinations of octahedra and tetrahedra. Many of these structures are compatible with an HCP lattice but not FCC. Our results also suggest that the curvature of the pair potential near the minimum should affect nucleation, because the curvature determines the free energy of the non-rigid clusters.

Structures with five-fold symmetry such as the pentagonal dipyramid and icosahedron are highly unfavorable in our system. Therefore we do not expect icosahedra or other clusters with five-fold symmetry to be a structural motif in attractive hard sphere gels or fluid cluster phases (28) where the attraction is short-ranged.

In conclusion, we find that the most stable small clusters of hard spheres with short-ranged attractions can be determined by geometrical rules: (1) rotational entropy favors structures with fewer symmetry elements; (2) vibrational entropy favors non-rigid clusters, which have half-octahedral substructures sharing at least one vertex; and (3) potential energy favors clusters with both octahedral and tetrahedral substructures, allowing them to have extra bonds.

Our picture of the free energy landscape is still incomplete. The qualitative features of the landscape are independent of temperature for our experimental system because the depletion interaction is fundamentally entropic (14). This will not be the case for other types of interactions, such as DNA-mediated attractions (29). Also, under non-equilibrium conditions we expect a different distribution of structures then the ones shown here. Finally, our model for the landscape does not account for 
energy barriers or the connectivity between minima. It will be interesting to see if further studies can explain the emergence of bulk crystallization or structural arrest in terms of these topographical features and their geometrical underpinnings.

\section{References and Notes}

1. F. C. Frank, Proc. R. Soc. London, Ser. A 215, 43 (1952).

2. F. H. Stillinger, T. A. Weber, Science 225, 983 (1984).

3. D. Nelson, F. Spaepen, Solid State Phys. 42, 1 (1989).

4. J. P. K. Doye, D. J. Wales, Science 271, 484 (1996).

5. H. Reichert, et al., Nature 408, 839 (2000).

6. H. W. Sheng, W. K. Luo, F. M. Alamgir, J. M. Bai, E. Ma, Nature 439, 419 (2006).

7. C. P. Royall, S. R. Williams, T. Ohtsuka, H. Tanaka, Nat. Mater. 7, 556 (2008).

8. M. Hoare, P. Pal, Adv. Phys. 24, 645 (1975).

9. M. Hoare, J. McInnes, Adv. Phys. 32, 791 (1983).

10. D. J. Wales, et al., Cambridge cluster database, http://wwwwales.ch.cam.ac.uk/CCD.html (2008).

11. D. J. Wales, T. V. Bogdan, J. Phys. Chem. B 110, 20765 (2006).

12. J. P. K. Doye, F. Calvo, J. Chem. Phys. 116, 8307 (2002).

13. R. L. Johnston, Atomic and molecular clusters (CRC Press, 2002).

14. Materials and methods are available as supporting material on Science Online.

15. S. Asakura, F. Oosawa, J. Chem. Phys. 22, 1255 (1954).

16. A. Vrij, Pure Appl. Chem. 48, 471 (1976).

17. V. Anderson, H. Lekkerkerker, Nature 416, 811 (2002).

18. N. Arkus, V. N. Manoharan, M. P. Brenner, Phys. Rev. Lett. 103, 118303 (2009).

19. M. Hoare, P. Pal, Adv. Phys. 20, 161 (1971).

20. C. Brooks, J. N. Onuchic, D. J. Wales, Science 293, 612 (2001). 
21. K. Dill, H. Chan, Nat. Struct. Biol. 4, 10 (1997).

22. Our particles are macroscopic and therefore distinguishable in principle. But we do not distinguish the particles in either the octahedral or polytetrahedral macrostate. The rotational partition function is therefore exactly the same as if we were to assume the particles to be indistinguishable.

23. G. Franke, E. R. Hilf, P. Borrmann, J. Chem. Phys. 98, 3496 (1993).

24. J. Doye, D. Wales, J. Chem. Soc. Faraday Trans. 93, 4233 (1997).

25. N. Johnson, Can. J. Math. 18, 169 (1966).

26. J. Bernal, Nature 185, 68 (1960).

27. V. Manoharan, M. Elsesser, D. Pine, Science 301, 483 (2003).

28. A. Stradner, et al., Nature 432, 492 (2004).

29. P. Biancaniello, A. Kim, J. Crocker, Phys. Rev. Lett. 94, 058302 (2005).

30. We thank Frans Spaepen and Zhengdong Cheng for helpful discussions. We acknowledge support from the National Science Foundation under award numbers DMR-0820484, CBET-0747625, and DMS-0907985 and DARPA under contract BAA 07-21.

\section{Supporting Online Material}

www.sciencemag.org

Materials and Methods

Figs. S1, S2

Tables S1, S2

Movies S1 to S9 


\title{
The Free Energy Landscape of Clusters of Attractive Hard Spheres
}

\author{
Supporting Online Material
}

\author{
Guangnan Meng, ${ }^{1}$ Natalie Arkus, ${ }^{2}$ Michael P. Brenner, ${ }^{2}$ Vinothan N. Manoharan ${ }^{1,2 *}$ \\ ${ }^{1}$ Department of Physics, Harvard University, Cambridge MA 02138 USA \\ ${ }^{2}$ Harvard School of Engineering and Applied Sciences, Harvard University, Cambridge MA 02138 USA \\ *To whom correspondence should be addressed; E-mail: vnm@ seas.harvard.edu
}

\section{Materials and Methods}

\section{Preparation of Particles, Microwells, and Clusters}

The model colloidal suspension we use consists of two types of particles, large polystyrene (PS) spheres, and small poly( $N$ '-Isopropylacrylamide) (poly(NIPAM)) spheres, which are used as a depletion agent to cause the larger spheres to attract one another. We synthesized the poly(NIPAM) spheres along with a third type of particle, poly( $N$ '-Isopropylacrylamide-co-Acrylic Acid) (poly(NIPAM-co-AAC)) particles, which were used to coat the microwells and prevent the large PS particles from sticking to them.

\subsection{Synthesis of poly(NIPAM) Hydrogel Particles}

We use precipitation polymerization to synthesize poly( $N$ '-Isopropylacrylamide) hydrogel particles. $2 \mathrm{~g}$ of $N$ '-Isopropylacrylamide (NIPAM, monomer, 99\%, Acros Organics), $25 \mathrm{mg} N, N$ '-Methylenebisacrylamide (crosslinker, 99\%, Promega), and $0.18 \mathrm{~g} \mathrm{(} \approx 6 \mathrm{mM})$ sodium dodecyl sulfate (SDS, 99\%, EMD Chemicals) were dissolved in $98 \mathrm{~mL}$ deionized (DI) water (Milli-Q synthesis grade, Millipore). The solution was stirred and heated to $70^{\circ} \mathrm{C}$ under nitrogen for 30 minutes to remove extra oxygen in the solution. $25 \mathrm{mg}$ of potassium persulfate (KPS, 99\%, Acros) were dissolved in $2 \mathrm{ml}$ DI water and loaded into the reactor to start the polymerization reaction. The reaction ran for 4 hours. Afterward, the poly(NIPAM) hydrogel particles were cleaned by dialysis against DI water for seven days, with water changes every 24 hours. We used a Zetasizer Nano ZS (Malvern Instruments) to measure the size of poly(NIPAM) hydrogel particles. The hydrodynamic diameter of our particles was $80 \mathrm{~nm}$ at $20^{\circ} \mathrm{C}$ and $40 \mathrm{~nm}$ at $40^{\circ} \mathrm{C}$ in DI water.

We synthesized a separate batch of poly( $N$ '-Isopropylacrylamide-co-Acrylic Acid) (poly(NIPAM-coAAc)) hydrogel particles to coat the microwell boundaries and glass substrate. This makes the microwell boundaries rough and prevents polystyrene particles from sticking to them through depletion interactions. The poly(NIPAM-co-AAc) hydrogel particles were synthesized using the same procedure as the $80 \mathrm{~nm}$ poly(NIPAM) particles, except that we added $25 \mathrm{mg}$ acrylic acid (99\%, Sigma) to the reactants.

\subsection{Fabrication and Surface Modification of Hydrogel Microwell Plates}

Microwell array plates were fabricated by soft lithography. As Fig. S1 shows, the surfaces of microwells and glass slides were then coated with a monolayer of $100 \mathrm{~nm}$ poly(NIPAM-co-AAc) hydrogel particles in 
order to prevent a depletion attraction between the PS particles and the boundaries. The fabrication involved the following steps:

1. Design of photomask: A photomask pattern was designed in AutoCAD (Autodesk Inc.). The pattern $(20 \mathrm{~mm} \times 20 \mathrm{~mm})$ has an array of circles $30 \mu \mathrm{m}$ in diameter with a pitch of $60 \mu \mathrm{m}$ on a square lattice. The pattern was printed on a photomask transparency at 20,000 dpi resolution by CAD/Art Services, Inc. (http://www. outputcity.com/)

2. Fabrication of SU-8 master mold. Under a clean room environment, a master mold of SU-8 photoresist (MicroChem Corp.) layer with the microwell pattern was fabricated on a silicon wafer by standard photolithography procedures (S1). The SU-8 3035 was spin-coated at $3000 \mathrm{rpm}$ onto a silicon wafer (University Wafer), setting the thickness of the SU-8 layer at $35 \mu \mathrm{m}$ (http: //www . microchem.com/ products/su_eight.htm).

3. Silanization of hydrogel-side cover slips and top glass slides: Precleaned No. 1 cover slips $(24 \mathrm{~mm} \times$ $30 \mathrm{~mm}, \mathrm{VWR}$ ) were silanized so that they could later be covalently bonded to the hydrogel. The same procedure was performed for precleaned glass slides $(25 \mathrm{~mm} \times 75 \mathrm{~mm}$, VWR), which were later bonded to poly(NIPAM-co-AAc) particles. Cover slips were immersed in $1.0 \% \mathrm{w} / \mathrm{w} 3-$ Methacryloxypropyltrimethoxysilane (98\%, Sigma) in anhydrous ethanol solution for 24 hours at room temperature. Glass slides were immersed in 1.0\% w/w (3-Aminopropyl)triethoxysilane (98\%, Sigma) in anhydrous ethanol solution for 24 hours at room temperature. Then the cover slips and glass slides were rinsed with anhydrous ethanol and blow-dried with compressed air. The silanization was completed by leaving the cover slips and glass slides in an oven at $110^{\circ} \mathrm{C}$ for one hour.

4. Hydrogel microwell fabrication: Pre-gel solution was prepared by dissolving $10 \% \mathrm{w} / \mathrm{w}$ acrylamide (99\%, Promega), 0.5\% w/w N,N'-Methylenebisacrylamide (crosslinker,99\%, Promega), 0.5\% w/w allylamine (98\%, Alfa Aesar) and 0.1\% w/w DAROCUR 1173 (photoinitiator, Ciba) in DI water. The pre-gel solution was poured onto the SU-8 master mold and covered by putting the silanized cover slip on top. The solution was placed $10 \mathrm{~cm}$ from an UV lamp (B-100YP, UVP) for 10 minutes to polymerize the hydrogel. The polymerized microwell plate was carefully separated from the SU-8 master mold, rinsed with DI water, and stored in DI water.

5. Surface coating of hydrogel microwell plates and glass slides: The surfaces of the hydrogel microwell plate and top glass slide were coated with a monolayer of $100 \mathrm{~nm}$ poly(NIPAM-co-AAc) hydrogel particles. The fabricated hydrogel microwell plate and silanized glass slides were immersed in a dialyzed colloidal suspension of $100 \mathrm{~nm}$ poly(NIPAM-co-AAc) particles for 24 hours at room temperature. Afterward the poly(NIPAM-co-AAc) hydrogel particles were irreversibly adsorbed onto the surfaces. After this surface treatment, we were able to form 3D colloidal clusters of PS spheres in the middle of the microwells. Without the surface treatment, PS spheres formed 2D crystallites on the boundaries of the microwells.

\subsection{Sample Preparation}

The aqueous colloidal suspension added to the microwells was a mixture of $1.0 \mu \mathrm{m}$ sulfate latex polystyrene (PS) particles (Batch\# 2090,1, Molecular Probes, polydispersity (standard deviation in particle diameter) $=3 \%$ ) and $80 \mathrm{~nm}$ poly(NIPAM) hydrogel particles in water. The concentration of PS was $10^{-3} \% \mathrm{w} / \mathrm{v}$ and poly(NIPAM) $1.0 \% \mathrm{w} / \mathrm{w}$ (the volume fraction is estimated to be $\phi_{s} \approx 25 \%$ at $20^{\circ} \mathrm{C}$ ). $15 \mathrm{mM} \mathrm{NaCl}$ were added to screen the long-ranged electrostatic repulsion between the PS particles. 0.1\% w/w Pluronic P123 (BASF) surfactant was also added to stabilize the PS particles in the salt. This procedure ensured that the 
depletion attraction between polystyrene particles induced from poly(NIPAM) could be reversed by diluting the poly(NIPAM) particles.

The hydrogel microwell plate and glass slides were rinsed with the PS/poly(NIPAM) suspension at least 5 times so that the hydrogel plate had the same ionic and surfactant concentration as the suspension. After the last rinse, the wells were filled with the suspension, and the microwell plate and glass slide were sealed with epoxy (Devcon 5-minute epoxy) around the edges of the cover slip. The number of particles $N$ per well were randomly distributed with a mean of about 10 . Before putting the sample on the optical microscope for observation and counting, we waited 24 hours for the system to reach equilibrium at $22.0 \pm 1.0^{\circ} \mathrm{C}$. We found that the hydrogel microwells tend to deform 10 days after sample preparation, so the observation and data collection was done within 7 days, and the sample was discarded afterward.

Under these experimental conditions, the colloidal clusters could transit between different mechanically stable configurations (Movie S1), demonstrating that the system is at equilibrium.

\section{Experimental Methods}

\subsection{Data Acquisition}

We used an inverted optical microscope (Eclipse TE-2000, Nikon Corp.) equipped a 100X objective (N.A.=1.4) and Nomarski differential interference contrast to observe the structures of the colloidal clusters. The images and videos $(720 \times 720,40$ frames per second) were digitally recorded by CCD cameras (Digital Sight DS-5Mc, Nikon Corp. for still images, and EO-0312C, Edmund Optics for movies) onto a personal computer. We scanned sequentially through the microwells and recorded videos of clusters in each before analyzing the data.

\subsection{Structure Identification}

We resolved the 3D structures of colloidal clusters by scanning through the recorded videos frame by frame. Although the microscope captures a 2D image with narrow depth of field, over time we can image all the particles in the cluster due to the Brownian rotational motion. We map out the nearest neighbors for each particle by looking at the 2D image and following it as the structure rotates in 3D space. We then compare this data to the contact matrices or computer renderings of different finite sphere packings identified in $(S 2)$.

\subsection{Counts and Error Analysis}

We calculated the probabilities of each structure from the number of occurrences in four different microwell arrays. To estimate the sampling error we used the Wilson score interval method (S3) (http: //faculty. vassar. edu/lowry/prop1.html) to estimate the lower and upper limits of the $95 \%$ confidence interval. For the small probabilities observed in our experiments, this method gives a much more accurate approximation of the confidence interval than a normal approximation to the multinomial distribution. The experimental statistics are shown in Table S1. Table S2 summarizes the experimental statistics of clusters for $9 \leq N \leq 12$.

\section{Theoretical Methods}

In the experiment, we measure the probability of structure $s$, where $s$ is determined by comparison to the sphere packings enumerated in (S2). In order to calculate the expected theoretical probability and other thermodynamic parameters for $s$, we start with the theoretically calculated coordinates for $s$. For each enumerated packing we know the equilibrium positions of all the particles, $\mathbf{r}_{i}^{0}=\left(x_{i}^{0}, y_{i}^{0}, z_{i}^{0}\right)$. With these 
coordinates, we calculate the potential energy $U_{s}$, the partition function $Z_{s}$, and the free energy $F_{s}$ using the methods below.

Calculations were done on an Intel-based desktop computer in the Python language (http://www . python.org) with the SciPy extensions (http://www.scipy.org).

\subsection{Pair Potential}

In our calculation of the potential energy we consider two major interactions between two PS particles, the depletion attraction $U_{A O}$ and an electrostatic repulsion $U_{D L V O} . U_{A O}$ was modeled by the Asakura-Oosawa depletion theory in a binary spherical mixture $(S 4-S 6)$ :

$$
U_{A O}(r)=-k_{B} T \frac{\phi_{s}}{\left(2 a_{s}\right)^{3}}\left(2 a_{s}+2 a_{l}-r\right)^{2} \times\left(2 a_{s}+2 a_{l}+\frac{r}{2}\right)
$$

where $r$ is the center-to-center distance between two PS particles, $\phi_{s}$ the volume fraction of poly(NIPAM) depletant $(\approx 25 \%)$, and $a_{l}$ and $a_{s}$ the radii of the large PS particles $(500 \mathrm{~nm})$ and small poly(NIPAM) (40 $\mathrm{nm}$ ) depletants. The range of the interaction is the diameter of the poly(NIPAM) depletant $2 a_{s}=80 \mathrm{~nm}$.

The surfaces of PS particles have negatively-charged sulfate groups. When two PS particles are very close to each other $\left(r_{i, j} \simeq 2 a_{l}=1.0 \mu \mathrm{m}\right)$, there is a screened Coulomb repulsion between them. This modifies the shape of the depletion well at small separation:

$$
U_{D L V O}(r)=k_{B} T Z^{2} \lambda_{B}\left(\frac{e^{\kappa a_{l}}}{1+\kappa a_{l}}\right)^{2} \frac{e^{-\kappa r}}{r}
$$

where $Z$ is surface charge valence per PS particle, $\lambda_{B}$ is Bjerrum length, $\kappa^{-1}$ is the Debye screen length (S7). For our experimental conditions, $Z=7.6 \times 10^{5}$ (from manufacturer), $\lambda_{B}=0.7 \mathrm{~nm}$, and $\kappa^{-1}=2 \mathrm{~nm}$.

The pair potential $U(r)$ we use for calculations is the summation of the depletion attraction and Coulomb repulsion:

$$
U(r)=U_{A O}(r)+U_{D L V O}(r) .
$$

The pair potential has a minimum at $1.02 d$, where $\mathrm{d}$ is the sphere diameter. We therefore normalize all distances in the problem to the position of the minimum. The calculated well depth is about $2 k_{B} T$. The true well depth in our system is probably closer to $4 k_{B} T$, as estimated from the typical time for cluster rearrangements, which is on the order of tens to hundreds of seconds. A better theoretical estimate of the well depth requires a measurement of the true surface charge of the particles. In calculating the free energy landscape (Figure 4), we chose $Z=6.4 \times 10^{4}$, which gives a potential depth of $4 k_{B} T$ at a distance of $1.005 d$. All other calculations used the parameters given above.

The attractive interaction is short-ranged compared to the PS particle diameter. Because the ratio of the large to small sphere diameters is large, the total potential energy of a colloidal cluster is pair-wise additive.

$$
U=\sum_{i, j} U\left(r_{i j}\right)=\sum_{i, j} U\left(\left|\mathbf{r}_{i}-\mathbf{r}_{j}\right|\right)
$$

The details of the potential have little effect on the calculated free energy except in two cases: non-rigid clusters and clusters derived from the pentagonal dipyramid. For non-rigid clusters the curvature of the well affects the integral in Eq. 13. For clusters with gaps within the range of the potential a small difference $\left(0.1 k_{B} T\right)$ in the magnitude of the potential at the gap distance can have a significant $(\approx 5 \%)$ effect on the summed probabilities in Table $\mathrm{S} 1$. 


\subsection{Free Energy, Entropy and Partition Function}

In the canonical ensemble $(N V T)$ ensemble, the probability $P_{s}$ of a state $s$ is given by the Boltzmann distribution:

$$
P_{s} \propto e^{-\beta F_{s}}=Z_{s} e^{-\beta U_{s}},
$$

where $Z_{s}$ is the partition function and $F_{s}$ is the Helmholtz free energy of state $s$. We calculate the internal partition function $Z_{s}$ and then use Eq. 5 to calculate the free energy $F_{s}$ of a structure $s(S 8)$.

The entropy of the cluster is proportional to the internal partition function $Z_{s}$. $Z_{s}$ can be written as the product of three approximately independent terms:

$$
Z_{s}=Z_{t, s} Z_{r, s} Z_{v, s}
$$

where $Z_{t, s}$ is the translational partition function, $Z_{r, s}$ the rotational partition function, and $Z_{v, s}$ the vibrational partition function for a colloidal cluster $s$.

The translational partition function is proportional to the free volume that the structure can explore. Because the volume of the microwell $\left(\approx(30 \mu \mathrm{m})^{3}=3 \times 10^{4} \mu \mathrm{m}^{3}\right)$ is much larger than the size of a cluster $\left(\approx 10 \mu \mathrm{m}^{3}\right)$, the free volume is approximately the same for all clusters, and the boundaries have no effect on the cluster configurations. Thus we assume $Z_{t, s}$ to be the same for all clusters.

\subsection{Rotational Partition Function}

The rotational partition function reflects the number of orientations and particle permutations of the cluster.

$$
Z_{r}=c_{r} \frac{\sqrt{I}}{\sigma},
$$

where $c_{r}$, a product of the temperature and fundamental constants, is the same for all clusters, $I$ the determinant of the moment of inertia tensor, and $\sigma$ the rotational symmetry number. We calculate $I$ from the coordinates $\mathbf{r}_{i}$ of the particles assuming the mass of each particle $m_{i}$ is the same. We normalize the masses to 1 for the calculations of $I$ shown in the paper.

To calculate $\sigma$ we use an algorithm for symmetry detection $(S 9, S 10)$. This algorithm identifies the point group, which also allows us to determine which structures are chiral. These correspond to clusters in $C_{1}$ or $C_{2}$ groups, with no $v$ or $h$ subgroups.

\subsection{Vibrational Partition Function}

To calculate the vibrational partition function we assume that the vibrations of each particle about its equilibrium position are harmonic. The total potential energy $U$ can be expanded around the equilibrium configuration:

$$
\begin{aligned}
U\left(\mathbf{r}_{i}\right) & =U\left(\mathbf{r}_{i}^{0}+\delta \mathbf{r}_{i}\right)=U^{0}+\sum_{i, j}^{3 N} \frac{\partial^{2} U}{\partial x_{i} \partial x_{j}} \delta x_{i} \delta x_{j}+O\left(x^{4}\right) \\
& \approx U^{0}+\frac{1}{2} \sum_{\alpha}^{3 N} k_{\alpha} q_{\alpha}^{2}
\end{aligned}
$$

where $x_{i}$ are the Cartesian coordinates of each individual particle $x_{i} \in\left\{x_{1}, y_{1}, z_{1}, x_{2}, \ldots z_{N}\right\}$ and $q_{\alpha}$ is the internal coordinate of the structure. 
The vibrational motions are separated into different non-interacting modes, the normal modes (S11). The normal mode spring constants $k_{\alpha}$ are calculated by numerically solving for the eigenvalues of the Hessian:

$$
\mathbf{H}_{i j}=\frac{\partial^{2} U}{\partial x_{i} \partial x_{j}} .
$$

The Hessian is calculated from numerical derivatives of the pair potential. $\mathbf{H}$ has $3 \mathrm{~N}$ eigenvalues, six of which are zero, corresponding to translational and rotational degrees of freedom. The other $3 N-6$ are the spring constants of the vibrational modes. A vibrational mode with eigenvalue $k_{\alpha}$ has vibrational partition function:

$$
Z_{v, \alpha} \propto \int_{-\infty}^{+\infty} e^{-\beta U\left(q_{\alpha}\right)} \mathrm{d} q_{\alpha}=\int_{-\infty}^{+\infty} e^{-\frac{1}{2} \beta k_{\alpha} q_{\alpha}^{2}}=\sqrt{\frac{2 \pi}{k_{\alpha}}}
$$

where $q_{\alpha}$ is the coordinate of the normal mode $\alpha$.

The vibrational partition function of a cluster is the product of the partition functions of each individual $Z_{v, \alpha}$ :

$$
Z_{v}=c_{v} \prod_{\alpha}^{3 N-6} \sqrt{\frac{2 \pi}{k_{\alpha}}}
$$

where $c_{v}$ is a constant that is the same for all clusters.

\subsection{Vibrational Partition Function for Non-Rigid Structures}

The normal mode analysis overestimates the partition function for a non-rigid structures because the nonrigid mode has a zero eigenvalue. To approximate the partition function for non-rigid structures we must first find the normal mode eigenvectors, then integrate the Boltzmann factor along the non-rigid mode eigenvector. Because the non-rigid mode has a zero-eigenvalue, we first "shift" the translational and rotational eigenvalues to a non-zero constant in order to keep these modes from mixing with the non-rigid mode (S12). We then solve for the $3 N-7$ normal mode eigenvalues and the null-space of the matrix (which contains only the non-rigid eigenvector) using singular value decomposition.

If the non-rigid mode has index $\alpha=0$, then its contribution to the partition function is:

$$
Z_{v, 0} \propto \int_{-\infty}^{+\infty} e^{-\beta U\left(q_{0}\right)} \mathrm{d} q_{0}
$$

where the integral is taken along the non-rigid eigenvector, and the potential energy is calculated numerically from the pair potential. To calculate the vibrational partition function we multiply the $3 N-7$ normal mode partition functions (Eq. 11) by the numerically integrated partition function for the non-rigid mode.

\subsection{Calculation of Probabilities and Free Energies}

We calculate the internal partition function up to a constant, $Z_{t} c_{r} c_{v}$ at a given particle number $N$. This constant is the same for all clusters, so it cancels when we compute the probability:

$$
P_{s}=\frac{Z_{s} e^{-\beta U_{s}}}{\sum P_{s}}
$$

Given the coordinates of individual particles $\mathbf{r}_{i}$, the internal partition function $Z_{s}$ is calculated from Eq. 6, and potential energy $U_{s}$ is calculated from Eq. 4 . We then use the Boltzmann distribution to calculate the free energy of structure $s$ :

$$
F_{s}=-k_{B} T \ln P_{s}-F^{0}
$$


where $F^{0}$ is the reference free energy for a given $N$. For each $N$ we use the highest free energy cluster as the reference state.

The depletion interaction is entropic in origin. Although we have chosen to include the depletion potential in the internal energy term $U$ of the free energy, it scales linearly with $T$, so that any ratio of the probabilities of two structures is also independent of temperature. 


\section{Figures and Tables}

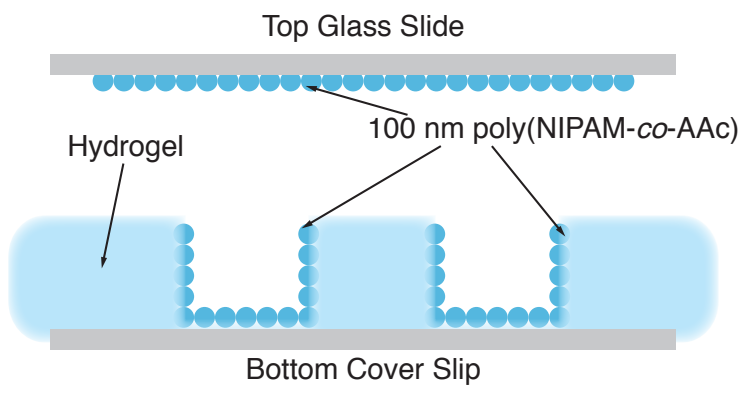

Fig. S1. Schematic view of surface treatment of microwells and glass slides. Boundaries of microwells are coated with monolayers of $100 \mathrm{~nm}$ poly(NIPAM-co-AAc) hydrogel particles to prevent a depletion attraction between the PS particles and planar surfaces. 


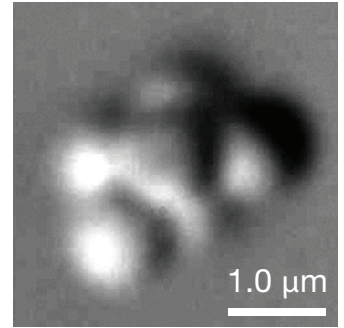

Polytetrahedron
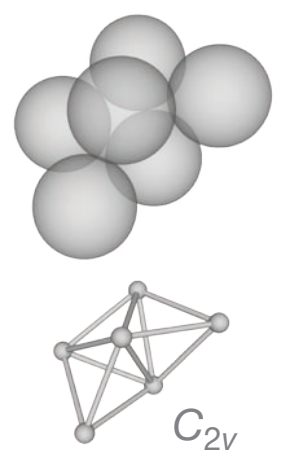

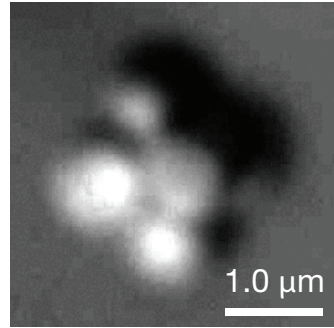

Octahedron
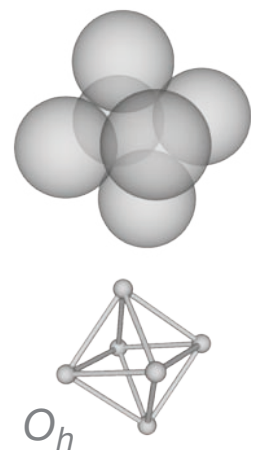

$\begin{array}{ccc}12 & \text { Contacts } & 12 \\ 2 & \text { Symmetry number } & 24 \\ 3.2 & \text { Moment of inertia }^{1 / 2} & 2.8 \\ 1.6 & Z_{\mathrm{r}} \text { (rotational) } & 0.12 \\ 0.061 & Z_{\mathrm{V}} \text { (vibrational) } & 0.034 \\ 96.0 \% & \text { Predicted } P & 4.0 \% \\ 95.7 \% & \text { Observed } P & 4.3 \%\end{array}$

Fig. S2. Comparison of polytetrahedral and octahedral clusters at $N=6$. Top shows optical micrographs and renderings of the two structures with point groups indicated in Schönflies notation, and bottom the entropic contributions to the probability of each. $Z_{r}$ is the rotational partition function and $Z_{v}$ the vibrational partition function. The predicted probability of formation is calculated from the product $Z_{v} Z_{r}$. The main contribution to the probability comes from the rotational partition function through the symmetry number. The calculated ratio of probabilities is $24: 1$, which agrees well with the experimental data (see also Table S1). 
Table S1. Number of observations and probabilities of clusters at $N=6,7$, and 8. "Graph \#" is a unique identifier for each cluster at each $N$, calculated from the contact graph generated by version 2.2 of the program NAUTY (http: //cs. anu . edu . au/ bdm/nauty/; see also (S2) for details of the sphere packing calculations). An asterisk (*) following the graph number indicates a chiral enantiomer. "Index" corresponds to the position of each cluster in the plots of Fig. 2. Index 1 corresponds to the leftmost cluster at each $N$. A $\Sigma$ symbol indicates a sum of probabilities of several similar structures, used to compare to experiment. "Point Group" is given in Schönflies notation. $\sigma$ is the rotational symmetry number of the cluster, and $P_{\text {theo }}$ is the theoretical probability, calculated as described in the theoretical methods section. "Counts" is the number of times a packing was observed experimentally. We use the Wilson score interval method to estimate the 95\% confidence intervals of the experimentally measured probabilities $P_{\exp }$.

\begin{tabular}{|c|c|c|c|c|c|c|c|}
\hline$N$ & Index & Graph \# & Point Group & $\sigma$ & $P_{\text {theo }}$ & Counts & $P_{\exp }(95 \%$ conf. inter.) \\
\hline \multirow[t]{2}{*}{6} & 1 & 2 & $C_{2 v}$ & 2 & $96.00 \%$ & 266 & $95.68 \%(92.38 \%-97.64 \%)$ \\
\hline & 2 & 4 & $O_{h}$ & 24 & $4.00 \%$ & 12 & $4.32 \%(2.27 \%-7.36 \%)$ \\
\hline \multirow[t]{7}{*}{7} & 1 & 4 & $C_{2 v}$ & 2 & $24.29 \%$ & & \\
\hline & 2 & 22 & $D_{5 h}$ & 10 & $2.83 \%$ & & \\
\hline & $\sum 1: 2$ & & & & $27.12 \%$ & 51 & $26.4 \%(20.5 \%-33.3 \%)$ \\
\hline & 3 & 8 & $C_{3 v}$ & 3 & $16.20 \%$ & 29 & $15.0 \%(10.5 \%-21.0 \%)$ \\
\hline & 4 & 17 & $C_{2}$ & 2 & $24.29 \%$ & 50 & $25.9 \%(20.0 \%-32.8 \%)$ \\
\hline & 5 & $17^{*}$ & $C_{2}$ & 2 & $24.29 \%$ & 48 & $24.9 \%(19.1 \%-31.7 \%)$ \\
\hline & 6 & 26 & $C_{3 v}$ & 3 & $8.10 \%$ & 15 & $7.8 \%(4.6 \%-12.7 \%)$ \\
\hline \multirow[t]{17}{*}{8} & 1 & 82 & $C_{1}$ & 1 & $9.28 \%$ & & \\
\hline & 2 & $82^{*}$ & $C_{1}$ & 1 & $9.28 \%$ & & \\
\hline & 3 & 77 & $C_{1}$ & 1 & $9.28 \%$ & & \\
\hline & 4 & $77^{*}$ & $C_{1}$ & 1 & $9.28 \%$ & & \\
\hline & 5 & 224 & $C_{s}$ & 1 & $8.94 \%$ & & \\
\hline & 6 & 271 & $C_{s}$ & 1 & $5.40 \%$ & & \\
\hline & $\sum 1: 6$ & & & & $51.44 \%$ & 76 & $66.7 \%(56.6 \%-74.5 \%)$ \\
\hline & 7 & 27 & $T_{d}$ & 12 & $0.77 \%$ & 0 & $0.0 \%(0.0 \%-4.0 \%)$ \\
\hline & 8 & 107 & $C_{s}$ & 1 & $9.28 \%$ & 7 & $6.1 \%(2.7 \%-12.6 \%)$ \\
\hline & 9 & 366 & $C_{s}$ & 1 & $9.28 \%$ & 12 & $10.5 \%(5.7 \%-17.9 \%)$ \\
\hline & 10 & 374 & $C_{1}$ & 1 & $9.28 \%$ & 7 & $6.1 \%(2.7 \%-12.6 \%)$ \\
\hline & 11 & $374^{*}$ & $C_{1}$ & 1 & $9.28 \%$ & 4 & $3.5 \%(1.1 \%-9.2 \%)$ \\
\hline & 12 & 204 & $C_{2 v}$ & 2 & $2.32 \%$ & 3 & $2.6 \%(0.7 \%-8.0 \%)$ \\
\hline & 13 & 294 & $C_{2 v}$ & 2 & $2.32 \%$ & 1 & $0.9 \%(0.05 \%-5.5 \%)$ \\
\hline & 14 & 397 & $D_{3 d}$ & 6 & $0.77 \%$ & 0 & $0.0 \%(0.0 \%-4.0 \%)$ \\
\hline & 15 & 358 & $C_{s}$ & 1 & $4.64 \%$ & 4 & $3.5 \%(1.1 \%-9.2 \%)$ \\
\hline & 16 & 408 & $D_{2 d}$ & 4 & $0.64 \%$ & 0 & $0.0 \%(0.0 \%-4.0 \%)$ \\
\hline
\end{tabular}


Table S2. Number of observations and probabilities of clusters for $9 \leq N \leq 12$. Clusters are categorized by number of bonds and rigidity. "Counts" is the number of times a packing was observed experimentally. We use the Wilson score interval method to estimate the $95 \%$ confidence intervals for the experimentally measured probabilities $P_{\text {exp. }}$.

\begin{tabular}{lclll}
\hline$N$ & Number of bonds & Rigidity & Counts & $P_{\exp }(95 \%$ conf. inter.) \\
\hline 9 & $21(3 N-6)$ & Non-Rigid & 4 & $11 \%(4 \%-27 \%)$ \\
& $21(3 N-6)$ & Rigid & 32 & $89 \%(73 \%-96 \%)$ \\
\hline 10 & $25(3 N-5)$ & Rigid & 4 & $12 \%(4 \%-29 \%)$ \\
& $24(3 N-6)$ & Non-Rigid & 7 & $21 \%(10 \%-39 \%)$ \\
& $24(3 N-6)$ & Rigid & 22 & $67 \%(48 \%-81 \%)$ \\
\hline 11 & $29(3 N-4)$ & Rigid & 2 & $7 \%(1 \%-24 \%)$ \\
& $28(3 N-5)$ & Rigid & 7 & $24 \%(11 \%-44 \%)$ \\
& $27(3 N-6)$ & Rigid & 20 & $69 \%(49 \%-84 \%)$ \\
\hline 12 & $33(3 N-3)$ & Rigid & 0 & $0 \%(0 \%-34 \%)$ \\
& $32(3 N-4)$ & Rigid & 1 & $10 \%(0.5 \%-46 \%)$ \\
& $31(3 N-5)$ & Rigid & 1 & $10 \%(0.5 \%-46 \%)$ \\
& $30(3 N-6)$ & Rigid & 8 & $80 \%(44 \%-96 \%)$ \\
\hline
\end{tabular}




\section{References}

S1. D. B. Weibel, W. R. DiLuzio, G. M. Whitesides, Nature Reviews Microbiology 5, 209 (2007).

S2. N. Arkus, V. N. Manoharan, M. P. Brenner, Phys. Rev. Lett. 103, 118303 (2009).

S3. R. Newcombe, Statistics In Medicine 17, 857 (1998).

S4. S. Asakura, F. Oosawa, J. Chem. Phys. 22, 1255 (1954).

S5. A. Vrij, Pure Appl. Chem. 48, 471 (1976).

S6. J. Crocker, J. Matteo, A. Dinsmore, A. Yodh, Phys. Rev. Lett. 82, 4352 (1999).

S7. W. B. Russel, D. A. Saville, W. R. Schowalter, Colloidal dispersions (Cambridge University Press, Cambridge, 1989).

S8. J. P. Sethna, Statistical mechanics: entropy, order parameters, and complexity, vol. 14 (Oxford University Press, Oxford, 2006).

S9. J. C. Cole, et al., Acta Crystallographica Sect. B 57, 88 (2001).

S10. T. W. Shattuck, ABC rotational constant calculator, http://www. colby. edu/chemistry/PChem/ scripts/ABC.html.

S11. E. Eyal, L. W. Yang, I. Bahar, Bioinformatics 22, 2619 (2006).

S12. R. E. Tuzun, D. W. Noid, B. G. Sumpter, C. Yang, Macromolecular Theory and Simulations 11, 711 (2002). 